\title{
Saphenous Vein Graft Aneurysms: A Growing Problem? Presentation of Two Cases and Surgical Management
}

\author{
Alicja Zientara ${ }^{1}$ Omer Dzemali ${ }^{1}$ Dragan Odavic ${ }^{1}$ Michele Genoni ${ }^{1}$ \\ ${ }^{1}$ Department of Cardiac Surgery, Stadtspital Triemli, Zürich, \\ Switzerland \\ Address for correspondence Alicja Zientara, Department of Cardiac \\ surgery, Stadtspital Triemli, Birmensdorferstrasse 497, Zürich 8063 , \\ Switzerland (e-mail: alicja_zientara@web.de). \\ Thorac Cardiovasc Surg Rep 2014;3:23-26.
}

\begin{abstract}
Keywords

- surgery

- complications

- reoperation

- off-pump surgery

- coronary artery bypass grafts surgery

- aneurysm

Background Saphenous vein graft aneurysm is a rare complication after coronary artery bypass grafting leading to reoperations.

Case Description In two patients with giant aneurysms, the subclavian artery and femoral vein were cannulated. The first patients' operation was combined with aortic valve replacement. The pulsating mass could be removed. The second patients' operation was planned as an off-pump coronary artery bypass grafting. Upon surgical entry, the aneurysm ruptured and the operation was continued as on-pump beating-heart procedure.

Conclusion Because of better follow-up and increasing use of diagnostics, more patients with graft aneurysms will be identified. The question about a treatment algorithm might be answered by aging population and improved survival.
\end{abstract}

\section{Introduction}

Saphenous vein graft aneurysms (SVGAs) are rare complications after coronary artery bypass grafting (CABG) and occur predominantly 10 to 20 years after the procedure at an estimated rate of $>1 \%{ }^{1}$ Regarding the etiology, different mechanisms including atherosclerotic degeneration, vessel wall ischemia, and changes in wall stress after the transposition of the vein into arterial circulation are discussed. ${ }^{2-4}$ The size of the aneurysm correlates with adverse events such as mechanical and bleeding complications. ${ }^{1}$ Till now, there exists no treatment algorithm for the therapy because of the rare occurrence. Although the first SVGA case was reported in 1975, more than one-third of the available reports were published after 2005. This increased reporting in recent years can be explained by higher life expectancy of CABG patients and increasing use of diagnostics such as computed tomography (CT), what emphasizes the importance of the topic. We present two patients with giant SVGAs as incidental findings, the following diagnostics, therapy concept, and operative management.

\section{Case Description}

Two patients (63 years [ - Fig. 1]; 71 years [ - Fig. 2]) presented with a giant SVGA in a CT (-Fig. 1b: $46 \times 78 \mathrm{~mm}$; - Fig. 2b: $98 \times 104 \mathrm{~mm}$ ). Both underwent a CABG 18 years before and had similar cardiovascular risk factors including arterial hypertension, dyslipidemia, and history of smoking.

The first patient suffered from dyspnea and angina pectoris under strain and at rest. The echocardiography revealed a new severe aortic valve stenosis (mean gradient: $47 \mathrm{~mm} \mathrm{Hg}$; valve orifice area: $\left.0.8 \mathrm{~cm}^{2}\right)$. A new stenosis of the large distal circumflex artery (CX) (90\%) and a functionally occluded and ectatic vein graft of unknown size to both marginal branches of the CX were diagnosed in the following angiography (-Fig. 1d,e). Furthermore, a good function of the left internal mammary artery (LIMA) to bypass the left anterior descending (LAD) artery and a chronic occlusion of another vein graft to the right coronary artery (RCA) with a good result of the proximal and medial stenting were found. From 2006 till 2008 , the patient had six percutaneous interventions because
License terms

Stuttgart · New York

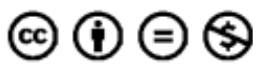

December 24, 2013 accepted after revision January 20, 2014 published online April 15, 2014
DOI http://dx.doi.org/ 10.1055/s-0034-1371700. ISSN 2194-7635. 


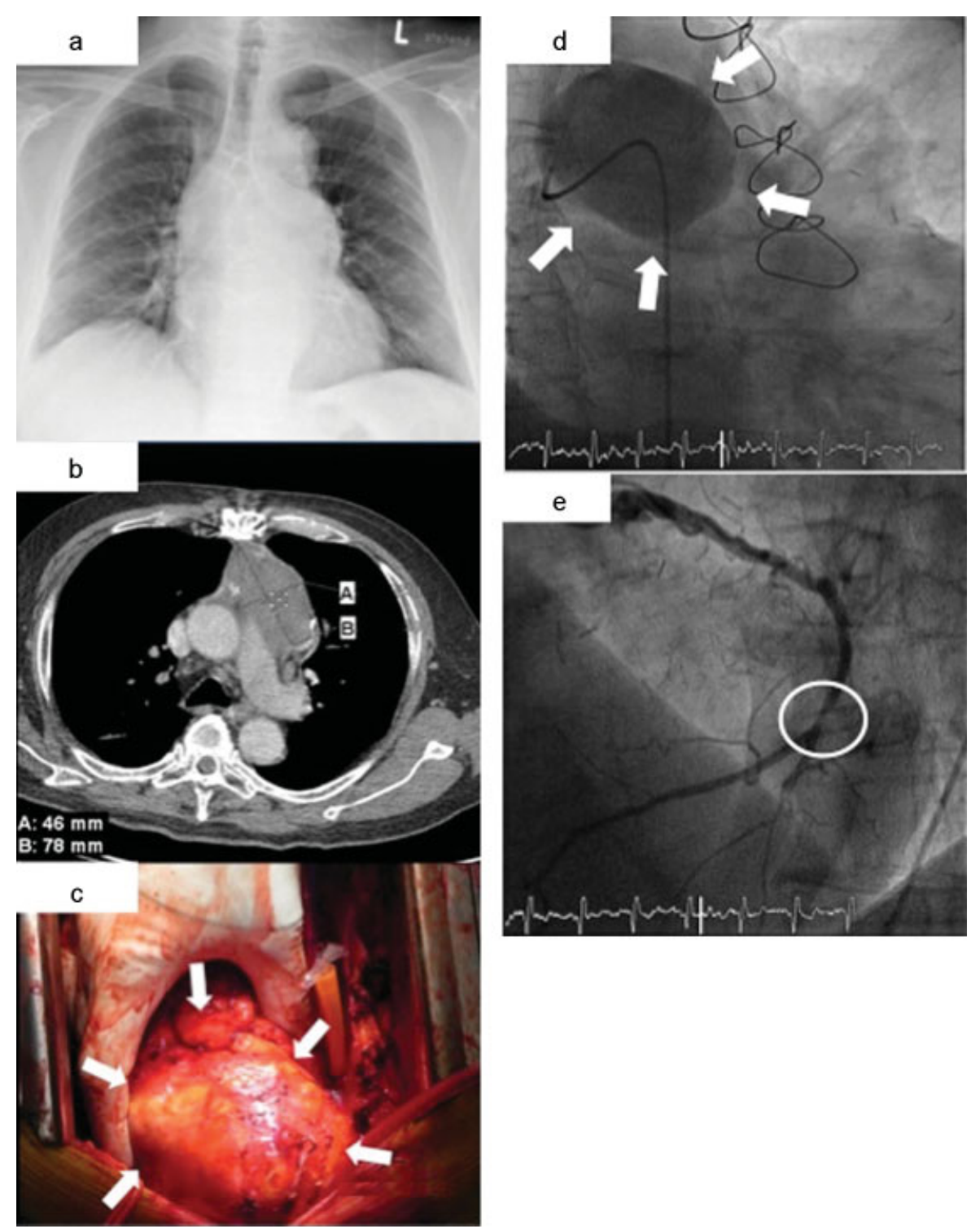

Fig. 1 (a) Chest X-ray (posteroanterior) with an enlargement of the mediastinum. (b) Venous graft to marginal branches of the circumflex artery. (c) White arrows show the dimension of the giant aneurysm, which lays directly beneath the sternum. (d) Perfused saphenous vein graft aneurysm to the circumflex artery (CX) in the angiography. (e) Distal stenosis of the large CX.

of repetitive angina pectoris including a stenting of the proximal CX and the RCA. Because of the distal stenosis of the large CX, severe aortic valve stenosis, and good general condition of the patient, we decided to perform a redooperation with bypass grafting to the $\mathrm{CX}$ and replacement of the aortic valve. To evaluate the size and localization of the ectatic vein before reoperation, a CT was performed showing the dimension of the SVGA $(46 \times 78 \mathrm{~mm})$ (-Fig. 1 b) directly localized behind the sternum neighboring the left pulmonary artery. Small parts in the thrombotic part of the aneurysms showed leakage of contrast.

The second patient had a CT because of hip pain revealing an infrarenal aneurysm. For evaluation of the thoracic aorta, another CT was performed showing a SVGA to the distal RCA $(98 \times 104 \mathrm{~mm})$ (-Fig. 2b). Concerning the priority of the following treatment, a coronary angiography was performed confirming the perfusion of the SVGA to the RCA with a stenosis of the distal anastomosis ( $\mathbf{- F i g . 2 d ) . ~ A ~ t o t a l ~ o c c l u s i o n ~ o f ~ a n o t h e r ~}$ graft to the marginal branch of the $\mathrm{CX}$ was found and a new stenosis of the first diagonal branch (-Fig. 2e). The LIMA showed a good function to the LAD. Due to the coronary disease and the huge, perfused SVGA, a CABG with resection of the SVGA was planned before the operation on the infrarenal aneurysm.

Surgical treatment: Before sternotomy, the right subclavian artery and the right femoral vein were cannulated in both patients as our standard strategy in redo-operation to prevent hemorrhage upon surgical entry.

In the first patient, the pulsating SVGA could be directly exposed and the cardiopulmonary bypass (CPB) started (-Fig. 1c). We prepared the patent LIMA-LAD bypass and the whole aneurysm neighboring the left pulmonary artery. Cross clamp of the aorta, antegrade and retrograde cardioplegia, and clamping of the LIMA-LAD bypass were performed. The aneurysm could be partially removed. Bypass 


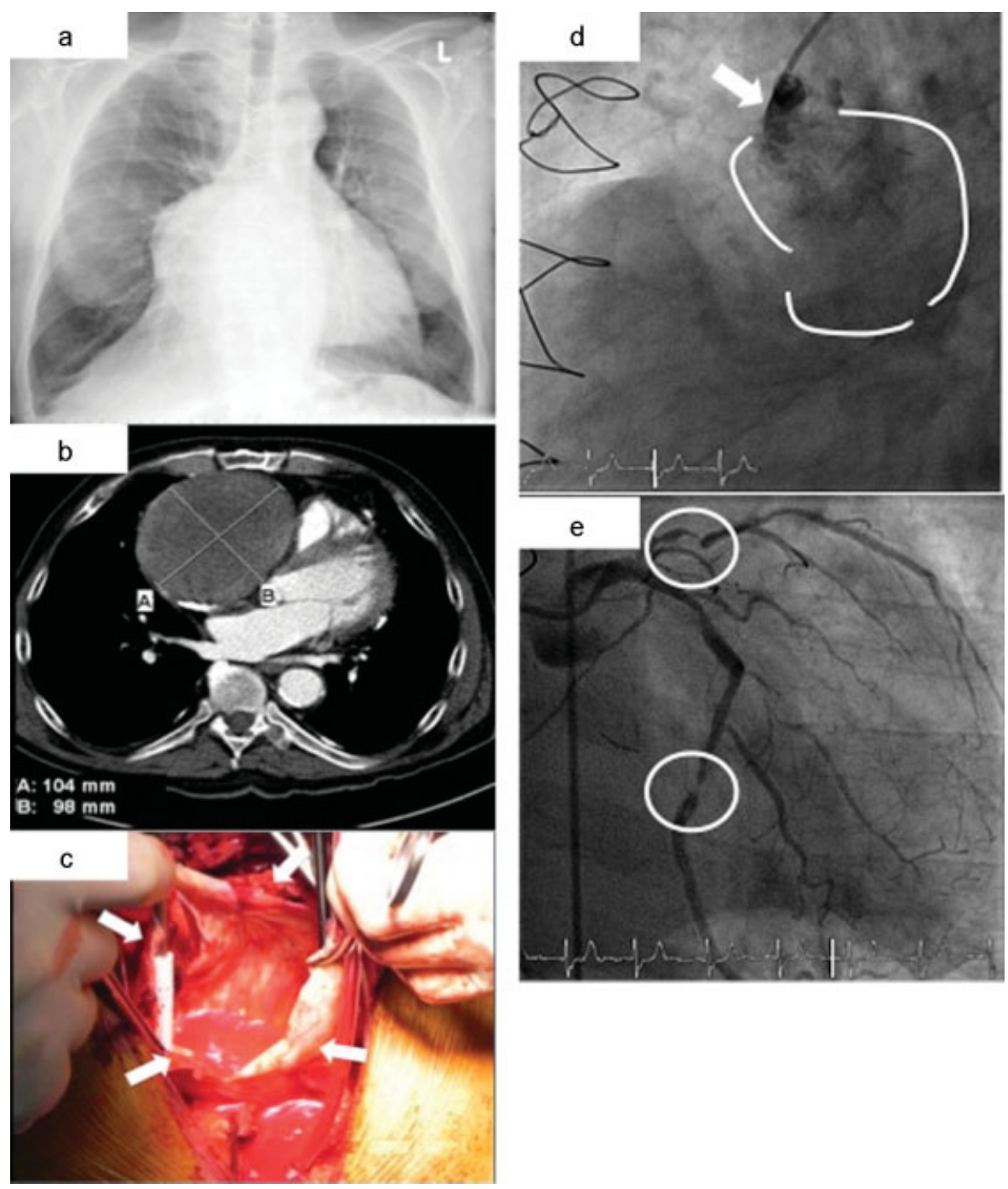

Fig. 2 (a) Chest X-ray (posteroanterior) with an enlargement of the mediastinum. (b) Venous graft to the right coronary artery. (c) The rupture of the aneurysm led to the decision to start with cardiopulmonary bypass to prevent hemorrhage. (d) Diffuse leakage of contrast demonstrates the partially perfused saphenous vein graft aneurysm. (e) Severe stenosis of the distal circumflex artery and the first diagonal branch.

to the distal CX and replacement of the aortic valve followed successfully.

In the second patient, the aneurysm ruptured (-Fig. 2c) during the preparation with minimal manipulation. CPB was started and the proximal part of the SVGA clamped and both ends sewn over. The operation was continued as an on-pump beating-heart procedure.

Postoperative course: The first patient arrived at the intensive care unit (ICU) with an open-chest treatment because of hemodynamic instability. The chest was closed the next day and the patient was extubated 3 days later. The second patient had an uneventful postoperative course at the ICU and on the normal ward. One week after the operation, an open-aortic repair with implantation of a Y-graft prosthesis was performed successfully.

\section{Discussion}

We present two uncommon cases of mediastinal mass (-Figs. 1a and 2a) revealing as giant SVGAs. Although the occurrence 18 years after the CABG is not uncommon, the localization next to complex structures and the huge diameter pose a challenge to the surgeon and raise questions concerning operative indication. Although a rupture as the most feared complication was reported very rarely, ${ }^{1}$ in both cases we decided for a redo-operation. In the first patient, the severe aortic valve stenosis required treatment. Without the repetitive angina pectoris and the new stenosis of the large CX, a treatment with transcatheter aortic valve implantation (TAVI) could be discussed. After the angiography and the CT with contrast leakage inside the aneurysm, the indication for operation was clarified. The second patient's aneurysm was partially perfused, too, which led to the decision of further investigations and revealed the coronary artery disease that was untreatable by stenting. However, with the tendency toward growth, there exists a positive relationship between SVGA size and adverse events resulting from mechanical complications.

It can be summarized that a detailed examination and diagnostics are mandatory for the decision of a therapy. Based on these findings, a reoperation or alternative treatment including TAVI or stenting can be discussed. Concerning the 
26 Saphenous Vein Graft Aneurysms Zientara et al.

further treatment, the perfusion of the aneurysm needs to be clarified. Perfused aneurysms should raise the awareness of future complications appearing with the growth. As shown by the second case, minimal manipulation of a perfused aneurysm can lead to massive bleeding. To reduce the risk of hemorrhage, we perform cannulation of the right subclavian artery and femoral vein as a standard strategy in redo-operations.

The increased reporting of SVGAs in recent years can be explained by higher life expectancy of CABG patients and increasing use of diagnostics. In future, however, an opposite trend can be expected due to the current strategy of complete arterial revascularization.

\section{Note}

This study was presented at the 27th Annual Meeting of the European Association for Cardio-Thoracic Surgery, Vienna, Austria, October 5 to 9, 2013.

\section{Conflict of Interest}

None declared.

\section{References}

1 Ramirez FD, Hibbert B, Simard T, et al. Natural history and management of aortocoronary saphenous vein graft aneurysms: a systematic review of published cases. Circulation 2012;126(18): 2248-2256

2 Memon AQ Huang RI, Marcus F, Xavier L, Alpert J. Saphenous vein graft aneurysm: case report and review. Cardiol Rev 2003;11(1): 26-34

3 Benchimol A, Harris CL, Desser KB, Fleming H. Aneurysms of an aorto-coronary artery saphenous vein bypass graft-a case report. Vasc Surg 1975;9(4):261-264

4 Vlodaver Z, Edwards JE. Pathologic changes in aorticcoronary arterial saphenous vein grafts. Circulation 1971;44(4): 719-728 